\title{
Positive solutions for singular nonlocal boundary value problems involving integral conditions with derivative dependence
}

\author{
Baoqiang Yan, Donal O'Regan and Ravi P. Agarwal
}

\begin{abstract}
In this paper using a fixed point theory on a cone we present some new results on the existence of multiple positive solutions for singular nonlocal boundary value problems involving integral conditions with derivative dependence.
\end{abstract}

\section{Introduction.}

In this paper we consider the existence of positive solutions of nonlinear nonlocal boundary value problems (BVP) of the form

$$
-x^{\prime \prime}=q(t) f\left(t, x(t), x^{\prime}(t)\right), t \in(0,1)
$$

with integral boundary conditions

$$
x^{\prime}(0)=0, x(1)=\alpha[x]=\int_{0}^{1} x(s) d A(s)
$$

involving a Stieltjes integral, where $A \in B V[0,1]$.

Il'in and Moiseev first considered the existence of a solution to

$$
x^{\prime \prime}(t)=f\left(t, x(t), x^{\prime}(t)\right), t \in(0,1),
$$

Key Words: Cone, compact operator, nonlocal boundary value problems.

2010 Mathematics Subject Classification: 34B10, 34B16.

Received: June, 2014.

Revised: July, 2014.

Accepted: July, 2014. 


$$
x(0)=0, x(1)=\sum_{i=1}^{m} \alpha_{i} x\left(\eta_{i}\right)
$$

(see $[10,11])$. Using degree-theoretic arguments, Gupta et al obtained conditions on the existence of solutions for the $m$-point boundary problem

$$
\begin{gathered}
x^{\prime \prime}(t)=f\left(t, x(t), x^{\prime}(t)\right)+e(t), t \in(0,1), \\
x(0)=0, x(1)=\sum_{i=1}^{m} \alpha_{i} x\left(\eta_{i}\right)
\end{gathered}
$$

(see $[7,8])$. In [12] using a Leray-Schauder alternative Ma showed the existence of at least one solution of

$$
\begin{gathered}
x^{\prime \prime}(t)=f\left(t, x(t), x^{\prime}(t)\right)+e(t), t \in(0,1), \\
x^{\prime}(0)=0, x(1)=\sum_{i=1}^{m} \alpha_{i} x\left(\eta_{i}\right) .
\end{gathered}
$$

In $[22,24]$, Webb and Infante considered

$$
-x^{\prime \prime}=q(t) f(t, x(t)), t \in(0,1)
$$

with boundary conditions

$$
x^{\prime}(0)=0, x(1)=\alpha[x]=\int_{0}^{1} x(s) d A(s),
$$

where $d A(s)$ has a signed measure, and established the existence of positive solutions and multiple positive solutions for BVP (1.3)-(1.4) when $f$ is continuous and independent of $x^{\prime}$.

The boundary condition in BVP (1.1)-(1.2) generalizes the boundary conditions in $[7-8,10-13]$ and (1.1) generalizes the equations in $[9,13-18,21-24]$ (there $f$ is independent of $x^{\prime}$ ). One goal in this paper is to attempt to fill a gap in the theory of singular nonlocal boundary value problems involving integral conditions with derivative dependence.

Our paper is organized as follows. In Section 2, we present some lemmas and preliminaries. In Section 3, two theorems are listed to show that $x^{\prime}$ of $f\left(t, x, x^{\prime}\right)$ can lead to BVP (1.1)-(1.2) having no positive solutions. In section 4 , we discuss the existence of multiple positive solutions for BVP (1.1)-(1.2) when $f$ has no singularities. Section 5 presents the multiplicity of positive solutions for BVP (1.1)-(1.2) when $f$ is singular at $x=0$ but not at $x^{\prime}=0$. In Section 6 , we discuss the multiplicity of positive solutions for BVP (1.1)-(1.2) when $f$ is singular at $x^{\prime}=0$ but not at $x=0$. In Section 7 , we consider the case $f$ is singular at $x=0$ and $x^{\prime}=0$. 


\section{Preliminaries}

Let $R=(-\infty,+\infty), R^{+}=(0,+\infty), \bar{R}^{+}=[0,+\infty), R^{-}=(-\infty, 0)$ and we list the following conditions for convenience.

$\left(C_{1}\right) A \in B V[0,1]$ with $\int_{0}^{1} G(t, s) d A(t) \geq 0$ for a.e. $s \in[0,1], 0 \leq \int_{0}^{1} d A(s)$ and $\int_{0}^{1}|d A(t)|<1$, where

$$
G(t, s)= \begin{cases}1-t, & 0 \leq s \leq t \leq 1 \\ 1-s, & 0 \leq t \leq s \leq 1\end{cases}
$$

$q \in C[0,1], q(t)>0$ on $(0,1)$ and $f \in C\left([0,1] \times R^{+} \times R^{-}, \bar{R}^{+}\right)$with $0<f(t, x, y) \leq[h(x)+w(x)][r(|y|)+v(|y|)]$ on $[0,1] \times R^{+} \times R^{-}$, where $w, v \in C\left(R^{+}, R^{+}\right)$are nonincreasing or $w \equiv 0, v \equiv 0$ and $h, r \in C\left(\bar{R}^{+}, \bar{R}^{+}\right)$are nondecreasing with $\int_{0}^{1} q(s) r\left(k_{0} \frac{1}{1-s}\right) d s<+\infty$, for all $k_{0}>0$,

$\left(C_{3}\right)$

$$
\begin{aligned}
& \text { there exists a constant } a \in\left(0, \frac{1}{2}\right) \text { such that } \\
& \lim _{x \rightarrow+\infty} \frac{f(t, x, y)}{x}=+\infty \\
& \text { uniformly for }(t, y) \in[a, 1-a] \times(-\infty, 0) .
\end{aligned}
$$

Let $p(t)=1-t, t \in[0,1]$ and $C_{p}^{1}[0,1]=\{x:[0,1] \rightarrow R \mid x$ is continuous on $[0,1]$ and continuously differentiable on $(0,1)$ with $\sup _{t \in(0,1)} p(t)\left|x^{\prime}(t)\right|=$ $\left.\sup _{t \in(0,1)}(1-t)\left|x^{\prime}(t)\right|<+\infty\right\}$. For $x \in C_{p}^{1}$, define $\|x\|=\max \left\{\|x\|_{1},\|x\|_{2}\right\}$ where

$\|x\|_{1}=\max _{t \in[0,1]}|x(t)|,\|x\|_{2}=\sup _{t \in(0,1)}(1-t)\left|x^{\prime}(t)\right|$.

Lemma 2.1 $C_{p}^{1}$ is a Banach space. Also for any $x \in C_{p}^{1},\left|x^{\prime}(t)\right| \leq \frac{\|x\|}{1-t}$, $t \in(0,1)$.

Let

$$
P=\left\{x \in C_{p}^{1} \mid x(t)\right. \text { is concave and nonincreasing on }
$$

$$
\left.[0,1] \text { and } x(0) \geq\|x\|_{2}, \alpha[x] \geq 0\right\} .
$$

It is easy to see that $P$ is a cone in $C_{p}^{1}[0,1]$.

We note the definition of the fixed point index $i(A, \Omega \cap P, P)$. Suppose that $\Omega$ is a bounded open set in real Banach space $E$ with $\theta \in \Omega$ as its vectoreal zero, $P$ is a cone of $E$ and $A: \bar{\Omega} \cap P \rightarrow P$ is continuous and compact. Assume that 
$r: E \rightarrow P$ is a retraction mapping, i.e., $r$ is continuous and $r(x)=x$ for all $x \in P$. Choose $R>0$ big enough such that $T_{R}=\{x \in E:\|x\|<R\} \supseteq \bar{\Omega} \cap P$. Then

$$
i(A, \Omega \cap P, P):=\operatorname{deg}\left(I-A \cdot r, T_{R} \cap r^{-1}(\Omega \cap P), \theta\right),
$$

where $I: E \rightarrow E$ is an identity operator and $\operatorname{deg}\left(\mathrm{I}-\mathrm{A} \cdot \mathrm{r}, \mathrm{T}_{\mathrm{R}} \cap \mathrm{r}^{-1}(\Omega \cap \mathrm{P}), \theta\right)$ is the Leray-Schauder degree (see [6]). The following lemmas are needed in Section 4-7.

Lemma 2.2(see[6]) Let $\Omega$ be a bounded open set in real Banach space $E$, $P$ be a cone of $E, \theta \in \Omega$ and $A: \bar{\Omega} \cap P \rightarrow P$ be continuous and compact. Suppose

$$
\lambda A x \neq x, \forall x \in \partial \Omega \cap P, \lambda \in(0,1] .
$$

Then

$$
i(A, \Omega \cap P, P)=1 \text {. }
$$

Lemma 2.3(see[6]) Let $\Omega$ be a bounded open set in real Banach space $E$, $P$ be a cone of $E, \theta \in \Omega$ and $A: \bar{\Omega} \cap P \rightarrow P$ be continuous and compact. Suppose

$$
A x \not \leq x, \forall x \in \partial \Omega \cap P .
$$

Then

$$
i(A, \Omega \cap P, P)=0 .
$$

Remark: $A x \not \leq x \Longleftrightarrow x-A x \notin P$.

Lemma 2.4 If $x \in P$ (defined above in (2.1)), then $\|x\|=\|x\|_{1}$.

Proof. If $x \in P$, one has

$$
\|x\|_{1}=\max \{|x(t)| t \in[0,1]\}=x(0) \geq\|x\|_{2} .
$$

Then

$$
\|x\|=\max \left\{\|x\|_{1},\|x\|_{2}\right\}=\|x\|_{1} .
$$

The proof is complete.

Lemma 2.5 Assume $\bar{\Phi} \in C\left((0,1), R^{+}\right)$with $\int_{0}^{1} \bar{\Phi}(t) d t<\infty$ and $F(t)=\int_{0}^{1} G(t, s) \bar{\Phi}(s) d s+c$, where $c \geq 0$ is a constant. Then $F \in P$. 
Proof. From the definition of $F$, we have

$$
\left\{\begin{array}{l}
F^{\prime \prime}(t) \leq 0, \quad t \in(0,1), \\
F^{\prime}(0)=0, \quad F(1)=c \geq 0,
\end{array}\right.
$$

which means that

$F$ is nonincreasing and concave down on $[0,1]$

with $F(1)=c \geq 0$. Now (2.2) implies that for $t \in[0,1], F(t)=F((1-t) 0+$ $t \cdot 1) \geq(1-t) F(0)+t F(1) \geq(1-t) F(0)=(1-t)\|F\|_{1}, \forall t \in(0,1)$ and

$$
F(0)=\max _{t \in[0,1]} F(t)=\|F\|_{1} .
$$

Then

$$
\begin{gathered}
\|F\|_{2}=\sup _{t \in(0,1)}\left|(1-t) F^{\prime}(t)\right| \\
=\sup _{t \in(0,1)}\left|(1-t) \int_{0}^{t} \bar{\Phi}(s) d s\right| \leq \sup _{t \in(0,1)}|F(t)|=\|F\|_{1}=F(0) .
\end{gathered}
$$

Moreover, from $\left(C_{1}\right)$, one has

$$
\begin{gathered}
\alpha[F]=c \int_{0}^{1} d A(s)+\int_{0}^{1} F(s) d A(s) \\
=c \int_{0}^{1} d A(s)+\int_{0}^{1} \bar{\Phi}(\tau) \int_{0}^{1} G(s, \tau) d A(s) d \tau \geq 0 .
\end{gathered}
$$

Hence, (2.2), (2.3) and (2.4) guarantee that $F \in P$. The proof is complete.

For $x \in P$, define an operator by

$$
(B x)(t)=\alpha[x]+\int_{0}^{1} G(t, s) q(s) f\left(s, x(s)+\gamma_{1}(s), x^{\prime}(s)+\gamma_{2}(s)\right) d s, \quad \forall t \in[0,1],
$$

where $\gamma_{1} \in C[0,1], \gamma_{2} \in C[0,1]$ with $\min _{t \in[0,1]} \gamma_{1}(t)>0$ and $\max _{t \in[0,1]} \gamma_{2}(t)<$ 0 .

Lemma 2.6 Assume that $\left(C_{1}\right)$ and $\left(C_{2}\right)$ hold. Then $B: P \rightarrow P$ is continuous and compact. 
Proof. First we show $B: P \rightarrow P$ is well defined. For $x \in P$, from $\left(C_{1}\right)$ and $\left(C_{2}\right)$, we have

$$
\begin{aligned}
&|(B x)(t)|=\left|\alpha[x]+\int_{0}^{1} G(t, s) q(s) f\left(s, x(s)+\gamma(s), x^{\prime}(s)+\gamma_{2}(s)\right) d s\right| \\
& \leq\|x\| \int_{0}^{1}|d A(s)|+\int_{0}^{1}(1-s) q(s)\left|f\left(s, x(s)+\gamma_{1}(s), x^{\prime}(s)+\gamma_{2}(s)\right)\right| d s \\
& \leq\|x\| \int_{0}^{1}|d A(s)|+\int_{0}^{1}(1-s) q(s)\left[h\left(x(s)+\gamma_{1}(s)\right)\right. \\
&\left.+w\left(x(s)+\gamma_{1}(s)\right)\right]\left[r\left(\left|x^{\prime}(s)+\gamma_{2}(s)\right|\right)+v\left(\left|x^{\prime}(s)+\gamma_{2}(s)\right|\right)\right] d s \\
& \leq\|x\| \int_{0}^{1}|d A(s)|+\int_{0}^{1}(1-s) q(s)\left[h\left(\|x\|+\left\|\gamma_{1}\right\|\right)+w\left(\min _{s \in[0,1]} \gamma_{1}(s)\right)\right] \\
& \cdot\left[r\left(\frac{\|x\|+\left\|\gamma_{2}\right\|}{1-s}\right)+v\left(\min _{s \in[0,1]}\left|\gamma_{2}(s)\right|\right)\right] d s<+\infty, \quad t \in[0,1]
\end{aligned}
$$

and

$$
\begin{aligned}
& (1-t)\left|(B x)^{\prime}(t)\right| \leq\left|(B x)^{\prime}(t)\right| \\
& =\left|\int_{0}^{t} q(s) f\left(s, x(s)+\gamma_{1}(s), x^{\prime}(s)+\gamma_{2}(s)\right) d s\right| \\
& \leq \int_{0}^{1} q(s)\left[h\left(\|x\|+\left\|\gamma_{1}\right\|\right)+w\left(\min _{s \in[0,1]} \gamma_{1}(s)\right)\right] \\
& \quad \cdot\left[r\left(\frac{\|x\|+\left\|\gamma_{2}\right\|}{1-s}\right)+v\left(\min _{s \in[0,1]}\left|\gamma_{2}(s)\right|\right)\right] d s<+\infty, \quad t \in[0,1] .
\end{aligned}
$$

Then $B$ is well defined. For every $x \in P$, let $\bar{\Phi}(t)=q(t) f\left(t, x(t)+\gamma_{1}(t), x^{\prime}(t)+\right.$ $\left.\gamma_{2}(t)\right), c=\alpha[x]$ and $F(t)=\int_{0}^{1} G(t, s) \bar{\Phi}(s) d s+c$. It is easy to see that all conditions of Lemma 2.5 hold, which implies that $B x \in P$. As a result, $B P \subseteq P$. Moreover, since

$$
\begin{aligned}
& \left|(B x)^{\prime}\left(t_{1}\right)-(B x)^{\prime}\left(t_{2}\right)\right| \\
= & \left|\int_{t_{1}}^{t_{2}} q(s) f\left(s, x(s)+\gamma_{1}(s), x^{\prime}(s)+\gamma_{2}(s)\right) d s\right| \\
\leq & \int_{t_{1}}^{t_{2}} q(s)\left[h\left(\|x\|+\left\|\gamma_{1}\right\|\right)+w\left(\min _{s \in[0,1]} \gamma_{1}(s)\right)\right] \\
& \cdot\left[r\left(\frac{\|x\|+\left\|\gamma_{2}\right\|}{1-s}\right)+v\left(\min _{s \in[0,1]}\left|\gamma_{2}(s)\right|\right)\right] d s,
\end{aligned}
$$

the Cauchy Principle guarantees that

$$
\lim _{t \rightarrow 0+}(B x)^{\prime}(t) \text { exists and } \lim _{t \rightarrow 1-}(B x)^{\prime}(t) \text { exists, }
$$

which means that $B x \in C^{1}[0,1]$. 
Next we show that $B: P \rightarrow P$ is continuous. Assume that $\left\{x_{m}\right\}_{m=1}^{\infty} \subseteq P$ and $x_{0} \in P$ with $\lim _{m \rightarrow+\infty} x_{m}=x_{0}$. Then, there exists an $M>0$ such that $\left\|x_{m}\right\|<M$ for all $m \in\{1,2, \cdots\}$ (Lemma 2.1 guarantees that $\left|x_{m}^{\prime}(t)\right| \leq \frac{M}{1-t}$, $\forall t \in(0,1))$. Thus,

$$
\begin{cases}\lim _{m \rightarrow+\infty}\left(x_{m}(t)+\gamma_{1}(t)\right)=x_{0}(t)+\gamma_{1}(t), & t \in[0,1] \\ \lim _{m \rightarrow+\infty}\left(x_{m}^{\prime}(t)+\gamma_{2}(t)\right)=x_{0}^{\prime}(t)+\gamma_{2}(t), & t \in(0,1)\end{cases}
$$

and

$$
\begin{aligned}
& \left|f\left(t, x_{m}(t)+\gamma_{1}(t), x_{m}^{\prime}(t)+\gamma_{2}(t)\right)\right| \\
& \leq\left[h\left(x_{m}(t)+\gamma_{1}(t)\right)+w\left(x_{m}(t)+\gamma_{1}(t)\right)\right]\left[r\left(\left|x_{m}^{\prime}(t)+\gamma_{2}(t)\right|\right)+v\left(\left|x_{m}^{\prime}(t)+\gamma_{2}(t)\right|\right)\right] \\
& \leq\left[h\left(M+\left\|\gamma_{1}\right\|\right)+w\left(\min _{t \in[0,1]} \gamma_{1}(t)\right)\right]\left[r\left(\frac{\|x\|+\left\|\gamma_{2}\right\|}{1-t}\right)+v\left(\min _{t \in[0,1]}\left|\gamma_{2}(t)\right|\right)\right] .
\end{aligned}
$$

From (2.6) and (2.7), the Lebesgue Dominated Convergence Theorem guarantees that

$$
\begin{aligned}
& \left\|B x_{m}-B x_{0}\right\|_{1} \\
& \leq\left|\alpha\left[x_{m}-x_{0}\right]\right|+\max _{t \in[0,1]} \mid \int_{0}^{1} G(t, s) q(s)\left[f\left(s, x_{m}(s)+\gamma_{1}(s), x_{m}^{\prime}(s)+\gamma_{2}(s)\right)\right. \\
& \left.\quad-f\left(s, x_{0}(s)+\gamma_{1}(s), x_{0}^{\prime}(s)+\gamma_{2}(s)\right)\right] d s \mid \\
& \leq\left\|x_{n}-x_{0}\right\| \int_{0}^{1}|d A(s)|+\int_{0}^{1}(1-s) q(s) \mid f\left(s, x_{m}(s)+\gamma_{2}(s), x_{m}^{\prime}(s)+\gamma_{2}(s)\right) \\
& \quad-f\left(s, x_{0}(s)+\gamma_{1}(s), x_{0}^{\prime}(s)+\gamma_{2}(s)\right) \mid d s \\
& \rightarrow 0, \text { as } m \rightarrow+\infty
\end{aligned}
$$

and

$$
\begin{aligned}
& \left\|B x_{m}-B x_{0}\right\|_{2} \\
= & \sup _{t \in(0,1)}(1-t) \mid-\int_{0}^{t} q(s)\left[f\left(s, x_{m}(s)+\gamma_{1}(s), x_{m}^{\prime}(s)+\gamma_{2}(s)\right)\right. \\
& \left.-f\left(s, x_{0}(s)+\gamma_{1}(s), x_{0}^{\prime}(s)+\gamma_{2}(s)\right)\right] d s \mid \\
\leq & \int_{0}^{1} q(s)\left|f\left(s, x_{m}(s)+\gamma_{1}(s), x_{m}^{\prime}(s)+\gamma_{1}(s)\right)-f\left(s, x_{0}(s)+\gamma_{1}(s), x_{0}^{\prime}(s)+\gamma_{2}(s)\right)\right| d s \\
& \rightarrow 0, \text { as } m \rightarrow+\infty,
\end{aligned}
$$

which imply that

$$
\lim _{m \rightarrow+\infty}\left\|B x_{m}-B x_{0}\right\|=0
$$

Hence, $B: P \rightarrow P$ is continuous.

Finally we show for any bounded $D \subseteq P, B(D)$ is relatively compact. Since $D$ is bounded, there exists an $M>0$ such that $\|x\| \leq M$ for all $x \in D$ 
(Lemma 2.1 guarantees that $\left.\left|x^{\prime}(t)\right| \leq \frac{M}{1-t}, \forall t \in(0,1)\right)$. Thus, $\left(C_{2}\right)$ gives

$$
\begin{aligned}
&\|B x\|_{1} \quad=\max _{t \in[0,1]}\left|\alpha[x]+\int_{0}^{1} G(t, s) q(s) f\left(s, x(s)+\gamma_{1}(s), x^{\prime}(s)+\gamma_{2}(s)\right) d s\right| \\
& \leq\|x\| \int_{0}^{1}|d A(s)|+\int_{0}^{1}(1-s) q(s)\left|f\left(s, x(s)+\gamma_{1}(s), x^{\prime}(s)+\gamma_{2}(s)\right)\right| d s \\
& \leq M \int_{0}^{1}|d A(s)|+\int_{0}^{1}(1-s) q(s)\left[h\left(x(s)+\gamma_{1}(s)\right)+w\left(x(s)+\gamma_{1}(s)\right)\right] \\
& \cdot\left[r\left(\left|x^{\prime}(s)+\gamma_{2}(s)\right|\right)+v\left(\left|x^{\prime}(s)+\gamma_{2}(s)\right|\right)\right] d s \\
& \leq M \int_{0}^{1}|d A(s)|+\int_{0}^{1}(1-s) q(s)\left[h\left(M+\left\|\gamma_{1}\right\|\right)+w\left(\min _{s \in[0,1]} \gamma_{1}(s)\right)\right] \\
& \cdot\left[r\left(\frac{M+\left\|\gamma_{2}\right\|}{1-s}\right)+v\left(\min _{s \in[0,1]}\left|\gamma_{2}(s)\right|\right)\right] d s
\end{aligned}
$$

and

$$
\begin{aligned}
& \sup _{t \in(0,1)}\left|(B x)^{\prime}(t)\right| \\
= & \sup _{t \in[0,1]}\left|-\int_{0}^{1} q(s) f\left(s, x(s)+\gamma_{1}(s), x^{\prime}(s)+\gamma_{2}(s)\right) d s\right| \\
\leq & \int_{0}^{1} q(s)\left|f\left(s, x(s)+\gamma_{1}(s), x^{\prime}(s)+\gamma_{2}(s)\right)\right| d s \\
\leq & \int_{0}^{1} q(s)\left[h\left(x(s)+\gamma_{1}(s)\right)+w\left(x(s)+\gamma_{1}(s)\right)\right] \\
& \cdot\left[r\left(\left|x^{\prime}(s)+\gamma_{2}(s)\right|\right)+v\left(\left|x^{\prime}(s)+\gamma_{2}(s)\right|\right)\right] d s \\
\leq & \int_{0}^{1} q(s)\left[h\left(M+\left\|\gamma_{1}\right\|\right)+w\left(\min _{s \in[0,1]} \gamma_{1}(s)\right)\right] \\
& \cdot\left[r\left(\frac{M+\left\|\gamma_{2}\right\|}{1-s}\right)+v\left(\min _{s \in[0,1]}\left|\gamma_{2}(s)\right|\right)\right] d s .
\end{aligned}
$$

Consequently,

the functions belonging to $\{(B D)(t)\}$ are uniformly bounded on $[0,1]$

and

the functions belonging to $\left\{(B D)^{\prime}(t)\right\}$ are uniformly bounded on $[0,1](2.9)$ and so

functions belonging to $\{(B x)(t), x \in D\}$ are equicontinuous on $[0,1]$. (2.10) 
Since

$$
\begin{aligned}
& \left|(B x)^{\prime}\left(t_{1}\right)-(B x)^{\prime}\left(t_{2}\right)\right| \\
& =\left|\int_{t_{1}}^{t_{2}} q(s) f\left(s, x(s)+\gamma_{1}(s), x^{\prime}(s)+\gamma_{1}(s)\right) d s\right| \\
& \leq \mid \int_{t_{1}}^{t_{2}} q(s)\left[h\left(M+\left\|\gamma_{1}\right\|\right)+w\left(\min _{s \in[0,1]} \gamma_{1}(s)\right)\right]\left[r\left(\frac{M+\left\|\gamma_{2}\right\|}{1-s}\right)+v\left(\min _{s \in[0,1]}\left|\gamma_{2}(s)\right|\right)\right] d s,
\end{aligned}
$$

for any $\varepsilon>0$, there exists a $\delta>0$ such that

$$
\left|(B x)^{\prime}\left(t_{1}\right)-(B x)^{\prime}\left(t_{2}\right)\right|<\varepsilon, \quad \forall\left|t_{1}-t_{2}\right|<\delta, \quad x \in D,
$$

which means that

the functions from $\left\{(B x)^{\prime}(t), x \in D\right\}$ are equicontinuous on $(0,1)$. (2.11)

Now $\{B x, x \in D\} \subseteq C^{1}[0,1]$, where $C^{1}[0,1]=\{y:[0,1] \rightarrow R: y(t)$ is continuously differentible on $[0,1]\}$ is a Banach space with norm $\|y\|_{0}=$ $\max \left\{\max _{t \in[0,1]}|y(t)|\right.$, $\left.\max _{t \in[0,1]}\left|y^{\prime}(t)\right|\right\}$.

From (2.8)-(2.11), the Arzela-Ascoli theorem guarantees that $B(D)$ is relatively compact in $C^{1}[0,1]$. Then, for any $\left\{x_{n}\right\} \subseteq D$, there exists a $y_{0} \in C^{1}[0,1]$ and a subsequence $\left\{x_{n_{i}}\right\}$ of $\left\{x_{n}\right\}$ such that

$$
\lim _{n_{i} \rightarrow+\infty}\left\|B x_{n_{i}}-y_{0}\right\|_{0}=0 .
$$

Now since

$$
\begin{gathered}
\left\|B x_{n_{1}}-y_{0}\right\| \\
=\max \left\{\max \left\{\left|\left(B x_{n_{i}}\right)(t)-y_{0}(t)\right| t \in[0,1]\right\}, \sup \left\{p(t)\left|\left(B x_{n_{i}}\right)^{\prime}(t)-y_{0}^{\prime}(t)\right| t \in(0,1)\right\}\right\} \\
\left.\leq \max \left\{\max \left\{\left|\left(B x_{n_{i}}\right)(t)-y_{0}(t)\right| t \in[0,1]\right\}, \max \left\{\left|\left(B x_{n_{i}}\right)^{\prime}(t)-y_{0}^{\prime}(t)\right| t \in[0,1]\right)\right\}\right\} \\
=\left\|B x_{n_{i}}-y_{0}\right\|_{0},
\end{gathered}
$$

we have

$$
\lim _{n_{i} \rightarrow+\infty}\left\|B x_{n_{i}}-y_{0}\right\|=0
$$

i.e., $B D$ is relatively compact in $C_{p}^{1}$.

Hence, $B: P \rightarrow P$ is continuous and compact.

Remark 1: We use the two functions $\gamma_{1} \in C[0,1], \gamma_{2} \in C[0,1]$ with $\min _{t \in[0,1]} \gamma_{1}(t)>0$ and $\max _{t \in[0,1]} \gamma_{2}(t)<0$ to help us remove the singularity of $f(t, x, y)$ at $x=0$ and $y=0$. If $f(t, x, y)$ is continuous at $x=0$ and $y=0$, we would take $\gamma_{1} \equiv 0, \gamma_{2} \equiv 0, t \in[0,1]$. 
Remark 2: Suppose that $x \in P$ satisfies $x=B x$, i.e.,

$$
x(t)=\alpha[x]+\int_{0}^{1} G(t, s) q(s) f\left(s, x(s)+\gamma_{1}(s), x^{\prime}(s)+\gamma_{2}(s)\right) d s, \quad \forall t \in[0,1] .
$$

Obviously, $x(1)=\alpha[x]$ and direct differentiating yields that

$$
x^{\prime}(t)=-\int_{0}^{t} q(s) f\left(s, x(s)+\gamma_{1}(s), x^{\prime}(s)+\gamma_{2}(s)\right) d s, t \in(0,1),
$$

which together with $q(s) f\left(s, x(s)+\gamma_{1}(s), x^{\prime}(s)+\gamma_{2}(s)\right) \in C[0,1]$ means that

$$
x^{\prime}(0)=0
$$

and

$$
x^{\prime \prime}(t)=-q(t) f\left(t, x(t)+\gamma_{1}(t), x^{\prime}(t)+\gamma_{2}(t)\right), t \in(0,1) .
$$

Hence, $x(t)$ satisfies

$$
x^{\prime \prime}(t)=-q(t) f\left(t, x(t)+\gamma_{1}(t), x^{\prime}(t)+\gamma_{2}(t)\right), t \in(0,1)
$$

with

$$
x^{\prime}(0)=0, \quad x(1)=\int_{0}^{1} x(s) d A(s)
$$

Lemma 2.7 Assume that $\left(C_{1}\right),\left(C_{2}\right)$ and $\left(C_{3}\right)$ hold. Then there exists $R_{0}>0$ such that

$$
i\left(B, \Omega_{R} \cap P, P\right)=0, \quad \forall R \geq R_{0},
$$

where $\Omega_{R}=\left\{x \in C_{p} \mid\|x\|<R\right\}$.

Proof. Let $N^{*}=\frac{2}{a \int_{a}^{1-a}(1-s) q(s) d s}$. From $\left(C_{3}\right)$, there exists $R^{\prime}>0$ such that

$$
f(t, x, y) \geq N^{*} x, \quad \forall x \geq R^{\prime}, y \in(-\infty, 0), t \in[a, 1-a] .
$$

Let $R_{0}=\frac{R^{\prime}}{a}$. For all $R>R_{0}$, set

$$
\Omega_{R}=\left\{x \in C_{p} \mid\|x\|<R\right\}
$$

Now we show that

$$
B x \not \leq x, \forall x \in P \cap \partial \Omega_{R} .
$$

In fact, suppose that there is a $x_{0} \in P \cap \partial \Omega_{R}$ with $B x_{0} \leq x_{0}$. Lemma 2.4 implies that $x_{0}(t) \geq(1-t)\left\|x_{0}\right\|, \forall t \in(0,1)$, and so $x_{0}(t) \geq a\left\|x_{0}\right\| \geq a R \geq R^{\prime}$ 
for all $t \in[a, 1-a]$. Thus, $x_{0}(t)+\gamma_{1}(t) \geq a\left\|x_{0}\right\| \geq a R \geq R^{\prime}$ for all $t \in[a, 1-a]$ also. Then, from (2.12), we have

$$
\begin{gathered}
f\left(t, x_{0}(t)+\gamma_{1}(t), x_{0}^{\prime}(t)+\gamma_{2}(t)\right) \geq N^{*}\left(x_{0}(t)+\gamma_{1}(t)\right) \\
\geq N^{*} x_{0}(t) \geq N^{*} a R, \quad \forall t \in[a, 1-a],
\end{gathered}
$$

and so

$$
\begin{aligned}
x_{0}(0) & \geq\left(B x_{0}\right)(0) \\
& =\alpha\left[x_{0}\right]+\int_{0}^{1}(1-s) q(s) f\left(s, x_{0}(s)+\gamma_{1}(s), x_{0}^{\prime}(s)+\gamma_{2}(s)\right) d s \\
& \geq \int_{a}^{1-a}(1-s) q(s) f\left(s, x_{0}(s)+\gamma_{1}(s), x_{0}^{\prime}(s)+\gamma_{2}(s)\right) d s \\
& \geq \int_{a}^{1-a}(1-s) q(s) N^{*}\left(x_{0}(s)+\gamma_{1}(s)\right) d s \\
& \geq \int_{a}^{1-a}(1-s) q(s) d s N^{*} a R \\
& >R
\end{aligned}
$$

which implies that $\left\|x_{0}\right\| \geq\left\|x_{0}\right\|_{1}>R$, a contradiction to $x_{0} \in P \cap \partial \Omega$. Then, (2.13) is true. From Lemma 2.3, it is easy to see that

$$
i(B, P \cap \Omega, P)=0 .
$$

The proof is complete.

\section{Nonexistence of positive solutions to $\operatorname{BVP}(1.1)-(1.2)$}

In this section, we notice that the presence of $z$ in $f(t, x, z)$ can lead to the nonexistence of positive solutions to (1.1)-(1.2).

Theorem 3.1 Suppose that there is a $\beta \in C((0,1),(0,+\infty))$ and $\delta>0$ such that

$$
f(t, y, z) \leq-\beta(t), \forall(t, y, z) \in(0,1) \times(0,+\infty) \times[-\delta, 0) .
$$

Then (1.1)-(1.2) has no positive solutions.

Proof. Suppose $y_{0}(t)$ is a positive solution to (1.1)-(1.2). Then

$$
\begin{gathered}
y_{0}^{\prime \prime}(t)+q(t) f\left(t, y_{0}(t), y_{0}^{\prime}(t)\right)=0, t \in(0,1) \\
y_{0}^{\prime}(0)=0, y_{0}(1)=\alpha[y]
\end{gathered}
$$


which means that there is a $t_{0} \in(0,1)$ with $y_{0}^{\prime}\left(t_{0}\right)<0, y_{0}\left(t_{0}\right)>0$ (otherwise $y^{\prime}(t) \geq 0$ for all $t \in(0,1)$ which would contradict $y(1)=\alpha[y] \leq$ $\left.\max _{t \in[0,1]} y(t) \int_{0}^{1}|d A(s)|<\max _{t \in[0,1]} y(t)\right)$. Let $t_{*}=\inf \left\{t<t_{0} \mid y_{0}^{\prime}(s)<0\right.$ for all $\left.s \in\left[t, t_{0}\right]\right\}$. Clearly,

$$
t_{*} \geq 0 \text { and } y_{0}^{\prime}\left(t_{*}\right)=0, y_{0}^{\prime}(t)<0 \text { for all } t \in\left(t_{*}, t_{0}\right] .
$$

The continuity of $y_{0}^{\prime}(t)$ implies that there is a $\gamma>0$ such that $0>y_{0}^{\prime}(t)>-\delta$ for all $t \in\left(t_{*}, t_{*}+\gamma\right]$. Then (3.1) guarantees that $f\left(t, y_{0}(t), y_{0}^{\prime}(t)\right) \leq-\beta(t)$ for all $t \in\left(t_{*}, t_{*}+\gamma\right]$, which implies that

$$
y_{0}^{\prime \prime}(t) \geq \beta(t)>0, \quad \forall t \in\left(t_{*}, t_{*}+\gamma\right]
$$

and so

$$
y_{0}^{\prime}(t)>0, \quad \forall t \in\left(t_{*}, t_{*}+\gamma\right],
$$

which contradicts $(3.2)$.

Consequently, (1.1)-(1.2) has no positive solutions.

Theorem 3.2 Suppose $q \in C[0,1]$ with $q(t)>0$ for all $t \in(0,1)$ and here are two functions $h \in C((0,+\infty),(0,+\infty)), g \in C((-\infty, 0),(0,+\infty))$ with

$$
\begin{aligned}
& |f(t, y, z)| \leq h(x) g(z), \forall(t, y, z) \in[0,1] \times(0,+\infty) \times(-\infty, 0), \\
& \text { where } \int_{z}^{0} \frac{1}{g(r)} d r=+\infty \text { for all } z<0 .
\end{aligned}
$$

Then (1.1)-(1.2) has no positive solutions.

Proof. Suppose $y_{0}(t)$ is a positive solution to (1.1)-(1.2). Then

$$
\begin{gathered}
y_{0}^{\prime \prime}(t)+q(t) f\left(t, y_{0}(t), y_{0}^{\prime}(t)\right)=0, t \in(0,1) \\
y_{0}^{\prime}(0)=0, y_{0}(1)=\alpha[y],
\end{gathered}
$$

which means that there is a $t_{0} \in(0,1)$ with $y_{0}^{\prime}\left(t_{0}\right)<0, y_{0}\left(t_{0}\right)>0$ (otherwise $y^{\prime}(t) \geq 0$ for all $t \in(0,1)$ which would contradict $y(1)=\alpha[y] \leq$ $\left.\max _{t \in[0,1]} y(t) \int_{0}^{1} \mid d A(s)<\max _{t \in[0,1]} y(t)\right)$. Let $t_{*}=\inf \left\{t<t_{0} \mid y_{0}^{\prime}(s)<0\right.$ for all $\left.s \in\left[t, t_{0}\right]\right\}$. Clearly,

$$
t_{*} \geq 0 \text { and } y_{0}^{\prime}\left(t_{*}\right)=0, y_{0}^{\prime}(t)<0 \text { for all } t \in\left(t_{*}, t_{0}\right] .
$$

Then, from (3.3),

$$
\begin{aligned}
-y_{0}^{\prime \prime}(t) & =q(t) f\left(t, y_{0}(t), y_{0}^{\prime}(t)\right) \\
& \leq q(t) h\left(y_{0}(t)\right) g\left(y_{0}^{\prime}(t)\right), t \in\left(t_{*}, t_{0}\right],
\end{aligned}
$$


and so

$$
-\frac{y_{0}^{\prime \prime}(t)}{g\left(y_{0}^{\prime}(t)\right)} \leq q(t) h\left(y_{0}(t)\right), t \in\left(t_{*}, t_{0}\right] .
$$

Integration from $t_{*}$ to $t_{0}$ yields

$$
+\infty=\int_{y_{0}^{\prime}\left(t_{0}\right)}^{0} \frac{1}{g(r)} d r \leq \int_{t_{*}}^{t_{0}} q(s) h\left(y_{0}(s)\right) d s<+\infty .
$$

This is a contradiction.

Consequently, (1.1)-(1.2) has no positive solutions.

Example 3.1. Consider the boundary value problems

$$
\begin{gathered}
x^{\prime \prime}+(1-t)^{a}\left[1-\left(\left|x^{\prime}\right|\right)^{-a}\right]\left[x^{b}+x^{-d}\right]=0, t \in(0,1), \\
x^{\prime}(0)=0, x(1)=\int_{0}^{1} x(s) d A(s),
\end{gathered}
$$

where $d A(t)=\frac{1}{8} \sin 2 \pi t d t, a>0, b>0, d>0$.

It is easy to see that $f(t, x, z)=(1-t)^{a}\left(1-(|z|)^{a}\right)\left[x^{b}+x^{-d}\right]$ for all $(t, x, z) \in$ $[0,1] \times[0,+\infty) \times(-\infty,+\infty)$. Since $\lim _{x \rightarrow 0+}\left(x^{b}+x^{-d}\right)=\lim _{x \rightarrow+\infty}\left(x^{b}+x^{-d}\right)=$ $+\infty$, there is a $c_{0}>0$ such that $x^{b}+x^{-d} \geq c_{0}$ for all $x \in(0,+\infty)$. Then $f(t, x, z) \leq(1-t)^{a}\left(1-2^{a}\right) c_{0}$ for all $(t, x, z) \in(0,1) \times(0,+\infty) \times\left[-\frac{1}{2}, 0\right)$. Then Theorem 3.1 guarantees that (3.5)-(3.6) has no positive solutions.

Example 3.2. Consider the boundary value problems

$$
\begin{gathered}
x^{\prime \prime}+\left(-x^{\prime}\right)^{a}\left[1+x^{b}\right]=0, \quad t \in(0,1), \\
x^{\prime}(0)=0, \quad x(1)=\int_{0}^{1} x(s) d A(s),
\end{gathered}
$$

where $d A(s)=\frac{1}{8} s d s, a>1, b>1, \mu>0, t \in[0,1]$.

Let $h(x)=1+x^{b}, g(y)=(-y)^{a}$. It is easy to see that

$$
|f(t, x, y)|=\left[1+x^{b}\right](-y)^{a}=h(x) g(y), \forall(t, x, y) \in[0,1] \times[0,+\infty) \times(-\infty, 0]
$$

and

$$
\int_{z}^{0} \frac{1}{g(y)} d y=+\infty, \forall z<0
$$

Theorem 3.2 implies that (3.7)-(3.8) has no positive solutions. 


\section{Multiple positive solutions to $\operatorname{BVP}(1.1)-(1.2)$ without singularities}

In this section, $f$ is continuous for $(t, x, y) \in[0,1] \times[0,+\infty) \times(-\infty, 0]$ and $c_{0}=\int_{0}^{1}|d A(s)|$.

Theorem 4.1 Suppose $\left(C_{1}\right)-\left(C_{3}\right)$ hold with $w(t) \equiv 0$ and $v(t) \equiv 0$ for all $t \in[0,+\infty)$ and

$$
\begin{aligned}
& \sup _{c \in(0,+\infty)} \frac{\left(1-c_{0}\right) c}{I^{-1}\left(h(c) \int_{0}^{1} q(s) d s\right)}>1, \\
& \text { where } I(z)=\int_{0}^{z} \frac{1}{r(u)} d u, z \in R^{+}, I(+\infty)=+\infty .
\end{aligned}
$$

Then (1.1) has at least two positive solutions $x_{0,1}, x_{0,2} \in C^{1}[0,1] \cap C^{2}(0,1)$ with $x_{0,1}(t) \geq 0$ and $x_{0,2}(t)>0$ on $(0,1)$.

Proof. From (4.1), choose an $R_{1}>0$ with

$$
\frac{R_{1}\left(1-c_{0}\right)}{I^{-1}\left(h\left(R_{1}\right) \int_{0}^{1} q(s) d s\right)}>1 .
$$

From $\left(C_{3}\right)$, choose an $R_{2}>\max \left\{R_{1}, \frac{R^{\prime}}{a}\right\}$ ( $R^{\prime}$ is defined as in (2.12)).

For $x \in P$, define

$$
(T x)(t)=\alpha[x]+\int_{0}^{1} G(t, s) q(s) f\left(s, x(s), x^{\prime}(s)\right) d s, \quad t \in[0,1] .
$$

It is easy to see that Lemma 2.6 guarantees that the operator $T$ in $(4.3)$ is continuous and compact from $P$ to $P$ (note here $\gamma_{1}(t) \equiv 0$ and $\gamma_{2}(t) \equiv 0$, for $t \in[0,1])$.

Let

$$
\Omega_{1}=\left\{x \in C_{p}^{1} \mid\|x\|<R_{1}\right\}
$$

and

$$
\Omega_{2}=\left\{x \in C_{p}^{1} \mid\|x\|<R_{2}\right\}
$$

Then, we claim that

$$
\mu T x \neq x, \quad \forall \mu \in(0,1], \quad x \in P \cap \partial \Omega_{1} .
$$

Now we show that (4.4) is true. Suppose there exists an $x_{0} \in P \cap \partial \Omega_{1}$ and a $\mu_{0} \in(0,1]$ such that $x_{0}=\mu_{0} T x_{0}$. Then

$$
\left\{\begin{array}{l}
x_{0}^{\prime \prime}(t)+\mu_{0} q(t) f\left(t, x_{0}(t), x_{0}^{\prime}(t)\right)=0, \quad t \in(0,1) \\
x_{0}^{\prime}(0)=0, \quad x_{0}(1)=\alpha\left[x_{0}\right]
\end{array}\right.
$$


which means that $x_{0}(t) \geq 0$ is on $(0,1)$ with $x_{0}^{\prime}(0)=0$ and $x_{0}^{\prime}(t)$ is nonincreasing on $(0,1)$. Without loss of generality, we assume that $x_{0}^{\prime}(t)<0$ for all $t \in(0,1)$ (obviously, $(4.7)$ is true for $\left.x_{0}^{\prime}(t)=0\right)$. From $(4.5)$, we have

$$
-x_{0}^{\prime \prime}(t) \leq q(t) f\left(t, x_{0}(t), x_{0}^{\prime}(t)\right) \leq q(t) h\left(x_{0}(t)\right) r\left(-x_{0}^{\prime}(t)\right), \quad \forall t \in(0,1),
$$

which means that

$$
\frac{-x_{0}^{\prime \prime}(t)}{r\left(-x_{0}^{\prime}(t)\right)} \leq h\left(x_{0}(t)\right) q(t) \leq h\left(R_{1}\right) q(t), \quad \forall t \in(0,1) .
$$

Integration from 0 to $t$ yields

$$
I\left(-x_{0}^{\prime}(t)\right)-I\left(-x_{0}^{\prime}(0)\right)=I\left(-x_{0}^{\prime}(t)\right) \leq h\left(R_{1}\right) \int_{0}^{1} q(s) d s
$$

and so

$$
-x_{0}^{\prime}(t) \leq I^{-1}\left(h\left(R_{1}\right) \int_{0}^{1} q(s) d s\right), \forall t \in(0,1) .
$$

Now integrate from 0 to 1 to obtain

$$
R_{1}\left(1-c_{0}\right) \leq x_{0}(0)-x_{0}(1) \leq I^{-1}\left(h\left(R_{1}\right) \int_{0}^{1} q(s) d s\right),
$$

a contradiction to (4.2). Then, (4.4) is true. Lemma 2.1 implies that

$$
i\left(T, \Omega_{1} \cap P, P\right)=1 \text {. }
$$

From Lemma 2.7, we have

$$
i\left(T, P \cap \Omega_{2}, P\right)=0,
$$

and so

$$
i\left(T, P \cap\left(\Omega_{2}-\bar{\Omega}_{1}\right), P\right)=-1 \text {. }
$$

As a result, there exist $x_{1} \in P \cap \Omega_{1}$ and $x_{2} \in P \cap\left(\Omega_{2}-\bar{\Omega}_{1}\right)$ such that $x_{1}=T x_{1}$ and $x_{2}=T x_{2}$.

Consequently, BVP(1.1)-(1-2) has at least two different nonnegative solutions $x_{1}(t)$ and $x_{2}(t)$ with $\left\|x_{1}\right\|<R_{1}<\left\|x_{2}\right\|$.

Example 4.1. Consider the boundary value problems

$$
x^{\prime \prime}+\mu\left[1+\left|x^{\prime}\right|^{a}\right]\left[1+x^{b}\right]=0, \quad t \in(0,1)
$$




$$
x^{\prime}(0)=0, \quad x(1)=\int_{0}^{1} x(s) d A(s),
$$

with $d A(t)=\frac{1}{8} \sin 2 \pi t d t, 0<a<1, b>1$ and $\mu>0$. If

$$
\mu<\sup _{c \in(0,+\infty)} \frac{\int_{0}^{\left(1-c_{0}\right) c} \frac{1}{1+s^{a}} d s}{1+c^{b}},
$$

then BVP(4.10)-(4.11) has at least two different positive solutions $x_{0,1}, x_{0,2} \in$ $C^{1}[0,1] \cap C^{2}(0,1)$.

It is easy to see that all conditions of Theorem 4.1 hold. Then, Theorem 4.1 guarantees that BVP(4.10)-(4.11) has at least two different positive solutions $x_{0,1}, x_{0,2} \in C^{1}[0,1] \cap C^{2}(0,1)$.

\section{Multiple positive solutions to $\operatorname{BVP}(1.1)-(1.2)$ with singularity at $x=0$ but not at $x^{\prime}=0$}

In this section our nonlinearity $f$ may be singular at $x=0$ but not at $x^{\prime}=0$ and $c_{0}=\int_{0}^{1}|d A(s)|$.

Theorem 5.1 Suppose $\left(C_{1}\right)-\left(C_{3}\right)$ hold with $w \in C((0,+\infty),(0,+\infty)) \cap$ $L_{l o c}[0,+\infty)$ and $v(t) \equiv 0$ for all $t \in[0,+\infty)$ and

$$
\begin{aligned}
& \sup _{c \in(0,+\infty)} \frac{\left(1-c_{0}\right) c}{I^{-1}\left(\|q\|_{0}\left[\operatorname{ch}(c)+\int_{0}^{c} w(s) d s\right]\right)}>1, \text { where } \\
& I(z)=\int_{0}^{z} \frac{u}{r(u)} d u, z \in(0,+\infty), I(+\infty)=+\infty,\|q\|_{0}=\max _{t \in[0,1]} q(t) .
\end{aligned}
$$

Then (1.1) has at least two positive solutions $x_{0,1}, x_{0,2} \in C^{1}[0,1] \cap C^{2}(0,1)$ with $x_{0,1}(t)>0$ and $x_{0,2}(t)>0$ on $(0,1)$.

Proof. From (5.1) and the continuity of $I^{-1}$ and $h$, choose an $R_{1}>0$, and a $\varepsilon>0$ with $\varepsilon<\frac{R_{1}}{2}$ with

$$
\frac{R_{1}\left(1-c_{0}\right)}{I^{-1}\left(\left(R_{1}+\varepsilon\right) h\left(R_{1}+\varepsilon\right)\|q\|_{0}+\|q\|_{0} \int_{0}^{R_{1}+\varepsilon} w(s) d s\right)}>1 .
$$

From $\left(C_{3}\right)$, choose a $R_{2}>\max \left\{R_{1}, \frac{R^{\prime}}{a}\right\}\left(R^{\prime}\right.$ is defined as in (2.12)). 
Let $n_{0} \in\{1,2, \cdots\}$ be chosen so that $\frac{1}{n_{0}}<\varepsilon$, and let $N_{0}=\left\{n_{0}, n_{0}+1, \cdots\right\}$. For each $n \in N_{0}$, for $x \in P$, define

$$
\left(T_{n} x\right)(t)=\alpha[x]+\int_{0}^{1} G(t, s) q(s) f\left(s, x(s)+\frac{1}{n}, x^{\prime}(s)\right) d s, \quad t \in[0,1] .
$$

Lemma 2.6 implies that $T_{n}: P \rightarrow P$ is continuous and compact (here $\gamma_{1}(t) \equiv \frac{1}{n}$ and $\gamma_{2}(t) \equiv 0$, for $\left.t \in[0,1]\right)$.

Let

$$
\Omega_{1}=\left\{x \in C_{q}^{1} \mid\|x\|<R_{1}\right\}
$$

and

$$
\Omega_{2}=\left\{x \in C_{q}^{1} \mid\|x\|<R_{2}\right\} .
$$

Then, for each $n \in N_{0}$, we claim that

$$
\mu T_{n} x \neq x, \quad \forall \mu \in(0,1], \quad x \in P \cap \partial \Omega_{1} .
$$

Now we show that (5.4) is true. Suppose there exists an $x_{0} \in P \cap \partial \Omega_{1}$ and a $\mu_{0} \in(0,1]$ such that $x_{0}=\mu_{0} T_{n} x_{0}$. Then

$$
\left\{\begin{array}{l}
x_{0}^{\prime \prime}(t)+\mu_{0} q(t) f\left(t, x_{0}(t)+\frac{1}{n}, x_{0}^{\prime}(t)\right)=0, \quad t \in(0,1) \\
x_{0}(0)=0, \quad x_{0}(1)=\alpha\left[x_{0}\right],
\end{array}\right.
$$

which means that $x_{0}(t)>0$ is on $(0,1)$ with $x_{0}^{\prime}(0)=0$ and $x_{0}^{\prime}(t)$ is nonincreasing on $(0,1)$. Without loss of generality, we assume that $x_{0}^{\prime}(t)<0$ for all $t \in(0,1)$ (obviously, $(5.7)$ is true for $x_{0}^{\prime}(t)=0$ ). From (5.5), we have

$$
\begin{aligned}
-x_{0}^{\prime \prime}(t) \leq q(t) f\left(t, x_{0}(t)+\frac{1}{n}, x_{0}^{\prime}(t)\right) & \leq q(t)\left[h\left(x_{0}(t)+\frac{1}{n}\right)+w\left(x_{0}(t)+\frac{1}{n}\right)\right] r\left(-x_{0}^{\prime}(t)\right), \\
& \forall t \in(0,1),
\end{aligned}
$$

which means that

$$
\frac{-x_{0}^{\prime \prime}(t)}{r\left(-x_{0}^{\prime}(t)\right)} \leq\left[h\left(x_{0}(t)+\frac{1}{n}\right)+w\left(x_{0}(t)+\frac{1}{n}\right)\right] q(t), \quad \forall t \in(0,1)
$$

and so

$$
\frac{x_{0}^{\prime}(t) x_{0}^{\prime \prime}(t)}{r\left(-x_{0}^{\prime}(t)\right)} \leq\left[h\left(x_{0}(t)+\frac{1}{n}\right)+w\left(x_{0}(t)+\frac{1}{n}\right)\right]\left(-x_{0}^{\prime}(t)\right) q(t), \quad \forall t \in(0,1) .
$$

Integration from 0 to $t$ yields

$$
I\left(-x_{0}^{\prime}(t)\right)-I\left(-x_{0}^{\prime}(0)\right)=I\left(-x_{0}^{\prime}(t)\right)
$$




$$
\leq\|q\|_{0} h\left(x_{0}(0)+\frac{1}{n}\right)\left(x_{0}(0)+\frac{1}{n}\right)+\|q\|_{0} \int_{\alpha\left[x_{0}\right]+\frac{1}{n}}^{x_{0}(0)+\frac{1}{n}} w(s) d s,
$$

and so

$$
-x_{0}^{\prime}(t) \leq I^{-1}\left(\|q\|_{0} h\left(R_{1}+\varepsilon\right)\left(R_{1}+\varepsilon\right)+\|q\|_{0} \int_{0}^{R_{1}+\varepsilon} w(s) d s\right), \forall t \in(0,1],
$$

Now integrate from 0 to 1 to obtain

$R_{1}\left(1-c_{0}\right) \leq x_{0}(0)-x_{0}(1) \leq I^{-1}\left(\|q\|_{0} h\left(R_{1}+\varepsilon\right)\left(R_{1}+\varepsilon\right)+\|q\|_{0} \int_{0}^{R_{1}+\varepsilon} w(s) d s\right)$,

a contradiction to (5.2). Then, (5.4) is true.

From Lemma 2.2, for each $n \in N_{0}$, we have

$$
i\left(T_{n}, P \cap \Omega_{1}, P\right)=1 \text {. }
$$

Lemma 2.7 implies that

$$
i\left(T_{n}, P \cap \Omega_{2}, P\right)=0,
$$

and so

$$
i\left(T_{n}, P \cap\left(\Omega_{2}-\bar{\Omega}_{1}\right), P\right)=-1, \quad n \in N_{0} .
$$

As a result, for each $n \in N_{0}$, there exist $x_{n, 1} \in P \cap \Omega_{1}$ and $x_{n, 2} \in P \cap\left(\Omega_{2}-\bar{\Omega}_{1}\right)$ such that $x_{n, 1}=T_{n} x_{n, 1}$ and $x_{n, 2}=T_{n} x_{n, 2}$.

Now we consider $\left\{x_{n, 1}\right\}_{n \in N_{0}}$ and $\left\{x_{n, 2}\right\}_{n \in N_{0}}$. Obviously, since $\left\{x_{n, 1}\right\}_{n \in N_{0}}$ is bounded, it is easy to see that

$$
\left\{x_{n, 1}(t)\right\} \text { is uniformly bounded on }[0,1]
$$

with $\max _{t \in[0,1]}\left|x_{n, 1}(t)\right| \leq R_{1}, n \in N_{0}$.

Using $x_{n, 1}$ instead of $x_{0}$ in (5.2), from (5.7), one has

$$
-x_{n, 1}^{\prime}(t) \leq I^{-1}\left(\|q\|_{0} h\left(R_{1}+\varepsilon\right)\left(R_{1}+\varepsilon\right)+\|q\|_{0} \int_{0}^{R_{1}+\varepsilon} w(s) d s\right), \forall t \in(0,1],
$$

which yields that

$$
\left\{x_{n, 1}^{\prime}(t)\right\} \text { is uniformly bounded on }[0,1]
$$

and so

$$
\left\{x_{n, 1}(t)\right\} \text { is equicontinous on }[0,1] \text {. }
$$


Using $x_{n, 1}$ instead of $x_{0}$ in (5.6), we have

$$
\frac{x_{n, 1}^{\prime}(t) x_{n, 1}^{\prime \prime}(t)}{r\left(-x_{n, 1}^{\prime}(t)\right)} \leq\left[h\left(x_{n, 1}(t)+\frac{1}{n}\right)+w\left(x_{n, 1}(t)+\frac{1}{n}\right)\right] q(t), \quad \forall t \in(0,1) .
$$

Integration from $t_{1}$ to $t_{2}$ yields

$$
\begin{aligned}
& \left.\mid I\left(-x_{n, 1}^{\prime}\left(t_{1}\right)\right)\right)-I\left(-x_{n, 1}^{\prime}\left(t_{2}\right)\right)|=| \int_{-x_{n, 1}\left(t_{1}\right)}^{-x_{n, 1}\left(t_{2}\right)} \frac{r}{g(r)} d r \mid \\
& \leq\left[h\left(R_{1}+\varepsilon\right)\left|x_{n, 1}\left(t_{1}\right)-x_{n, 1}\left(t_{2}\right)\right|+\left|\int_{-x_{n, 1}\left(t_{1}\right)+\frac{1}{n}}^{-x_{n, 1}\left(t_{2}\right)+\frac{1}{n}} w(r) d r\right|\right]\|q\|_{0} .
\end{aligned}
$$

Since $w \in L_{l o c}[0,+\infty)$, from $(5.12)$, we have

$$
\left\{I\left(-x_{n, 1}^{\prime}(t)\right)\right\} \text { is equicontinous on }[0,1] \text {. }
$$

Since

$$
\left|x_{n, 1}^{\prime}\left(t_{1}\right)-x_{n, 1}^{\prime}\left(t_{2}\right)\right|=\left|I^{-1}\left(I\left(-x_{n, 1}^{\prime}\left(t_{1}\right)\right)\right)-I^{-1}\left(I\left(-x_{n, 1}^{\prime}\left(t_{2}\right)\right)\right)\right|
$$

and $I^{-1}$ is uniformly continuous on $\left[0, I\left(R_{1}\right)\right]$, we have

$$
\left\{x_{n, 1}^{\prime}(t)\right\} \text { is equicontinuous on }[0,1] \text {. }
$$

From (5.10), (5.11), (5.12) and (5.14), the Arzela-Ascoli Theorem guarantees the existence of a subsequence $N=\left(n_{j}\right)$ of $N_{0}$ and a function $x_{0,1} \in$ $C^{1}[0,1]$ with $\lim _{j \rightarrow+\infty} x_{n_{j}, 1} \rightarrow x_{0,1}$ with

$$
x_{0,1}^{\prime}(0)=0 \text {. }
$$

From

$$
\begin{gathered}
\lim _{j \rightarrow+\infty} f\left(t, x_{n_{j}, 1}(t)+\frac{1}{n_{j}}, x_{n_{j}, 1}^{\prime}(t)\right)=f\left(t, x_{0,1}(t), x_{0,1}^{\prime}(t)\right), \quad \forall t \in(0,1), \\
x_{n_{j}, 1}(t)=x_{n_{j}, 1}\left(\frac{1}{2}\right)+x_{n_{j}, 1}^{\prime}\left(\frac{1}{2}\right)\left(t-\frac{1}{2}\right) \\
+\int_{\frac{1}{2}}^{t}(s-t) q(s) f\left(s, x_{n_{j}, 1}(s)+\frac{1}{n_{j}}, x_{n_{j}, 1}^{\prime}(s)\right) d s
\end{gathered}
$$

for $t \in(0,1)$ and

$$
x_{n_{j}, 1}(1)=\int_{0}^{1} x_{n_{j}, 1}(s) d A(s)
$$


the Lebesgue Dominated Convergence Theorem guarantees that

$$
\begin{gathered}
x_{0,1}(t)=x_{0,1}\left(\frac{1}{2}\right)+x_{0,1}^{\prime}\left(\frac{1}{2}\right)\left(t-\frac{1}{2}\right)+\int_{\frac{1}{2}}^{t}(s-t) q(s) f\left(s, x_{0,1}(s), x_{0,1}^{\prime}(s)\right) d s \\
x_{0,1}(1)=\int_{0}^{1} x_{0,1}(s) d A(s)=\alpha\left[x_{0,1}\right] .
\end{gathered}
$$

Hence, from (5.15)-(5.17), $x_{0,1}(t)$ is a positive solution to $\operatorname{BVP}(1.1)-(1.2)$ with $\left\|x_{0,1}\right\| \leq R_{1}$. Also (5.2) guarantees that $\left\|x_{0,1}\right\|<R_{1}$.

For the set $\left\{x_{n, 2}\right\}_{n \in N_{0}} \subseteq\left(\Omega_{2}-\bar{\Omega}_{1}\right) \cap P$, from the same proof for the set $\left\{x_{n, 1}\right\}_{n \in N_{0}}$, we can obtain a convergent subsequence $\left\{x_{n_{i}, 2}\right\}$ of $\left\{x_{n, 2}\right\}$ with $\lim _{i \rightarrow+\infty} x_{n_{i}, 2}=x_{0,2} \in C^{1}$. Moreover, $x_{0,2}$ is a positive solution to equation (1.1) with $R_{1}<\left\|x_{0,2}\right\|<R_{2}$.

Consequently, BVP(1.1)-(1.2) has at least two different positive solutions $x_{0,1}(t)$ and $x_{0,2}(t)$ with $\left\|x_{0,1}\right\|<R_{1}<\left\|x_{0,2}\right\|$.

Example 5.1. Consider the boundary value problems

$$
\begin{gathered}
x^{\prime \prime}+\mu\left[1+\left|x^{\prime}\right|^{a}\right]\left[1+x^{b}+x^{-d}\right]=0, \quad t \in(0,1) \\
x^{\prime}(0)=0, \quad x(1)=\int_{0}^{1} x(s) d A(s),
\end{gathered}
$$

with $d A(t)=\frac{1}{8} \sin 2 \pi t d t, 0<a<1, b>1,0<d<1$ and $\mu>0$. If

$$
\mu<\sup _{c \in(0,+\infty)} \frac{\int_{0}^{\left(1-c_{0}\right) c} \frac{s}{1+s^{a}} d s}{c+c^{b+1}+\frac{1}{1-d} c^{1-d}},
$$

then BVP(5.18)-(5.19) has at least two different positive solutions $x_{0,1}, x_{0,2} \in$ $C^{1}[0,1] \cap C^{2}(0,1)$.

It is easy to see that all conditions of Theorem 5.1 hold. Then, Theorem 5.1 guarantees that $\operatorname{BVP}(5.18)-(5.19)$ has at least two different positive solutions $x_{0,1}, x_{0,2} \in C^{1}[0,1] \cap C^{2}(0,1)$.

\section{Multiple positive solutions to $\operatorname{BVP}(1.1)-(1.2)$ with singularity at $x^{\prime}=0$ but not at $x=0$}

In this section our nonlinearity $f$ may be singular at $x^{\prime}=0$ but not $x=0$ and $c_{0}=\int_{0}^{1}|d A(s)|$. 
Theorem 6.1 Suppose $\left(C_{1}\right)-\left(C_{3}\right)$ hold with $v \in C((0,+\infty),(0,+\infty))$ and $w(t) \equiv 0$ for all $t \in[0,+\infty)$ and

$$
\begin{aligned}
& \sup _{c \in(0,+\infty)} \frac{\left(1-c_{0}\right) c}{I^{-1}\left(h(c) \int_{0}^{1} q(s) d s\right)}>1, \text { where } \\
& I(z)=\int_{0}^{z} \frac{1}{r(u)+v(u)} d u, z \in(0,+\infty), I(+\infty)=+\infty .
\end{aligned}
$$

Then $B V P(1.1)-(1.2)$ has at least two positive solutions $x_{0,1}, x_{0,2} \in C^{1}[0,1] \cap$ $C^{2}(0,1)$ with $x_{0,1}(t) \geq 0$ and $x_{0,2}(t)>0$ on $(0,1)$.

Proof. From (6.1) and the continuity of $I^{-1}$ and $h$, choose an $R_{1}>0$, and a $\varepsilon>0$ with $\varepsilon<\frac{R_{1}}{2}$ with

$$
\frac{R_{1}\left(1-c_{0}\right)}{I^{-1}\left(I(\varepsilon)+h\left(R_{1}\right) \int_{0}^{1} q(s) d s\right)}>1 .
$$

From $\left(C_{3}\right)$, choose a $R_{2}>\max \left\{R_{1}, \frac{R^{\prime}}{a}\right\}\left(R^{\prime}\right.$ is defined as in (2.12)).

Let $n_{0} \in\{1,2, \cdots\}$ be chosen so that $\frac{1}{n_{0}}<\varepsilon$, and let $N_{0}=\left\{n_{0}, n_{0}+1, \cdots\right\}$. For each $n \in N_{0}$, for $x \in P$, define

$$
\left(T_{n} x\right)(t)=\alpha[x]+\int_{0}^{1} G(t, s) q(s) f\left(s, x(s), x^{\prime}(s)-\frac{1}{n}\right) d s, \quad t \in[0,1] .
$$

Lemma 2.6 guarantees that for each $n \in N_{0}, T_{n}: P \rightarrow P$ is continuous and compact (here $\gamma_{1}(t) \equiv 0$ and $\gamma_{2}(t) \equiv-\frac{1}{n}$, for $\left.t \in[0,1]\right)$.

Let

$$
\Omega_{1}=\left\{x \in C_{q}^{1} \mid\|x\|<R_{1}\right\}
$$

and

$$
\Omega_{2}=\left\{x \in C_{q}^{1} \mid\|x\|<R_{2}\right\} .
$$

An argument similar to that in the proof of (5.4) shows that for each $n \in N_{0}$, we have that

$$
\mu T_{n} x \neq x, \quad \forall \mu \in(0,1], \quad x \in P \cap \partial \Omega_{1},
$$

which together with Lemma 2.2 implies

$$
i\left(T_{n}, P \cap \Omega_{1}, P\right)=1, n \in N_{0} .
$$

Since Lemma 2.7 guarantees that

$$
i\left(T_{n}, P \cap \Omega_{2}, P\right)=0, n \in N_{0}
$$


we have

$$
i\left(T_{n}, P \cap\left(\Omega_{2}-\bar{\Omega}_{1}\right), P\right)=-1, \quad n \in N_{0} .
$$

As a result, for each $n \in N_{0}$, there exist $x_{n, 1} \in P \cap \Omega_{1}$ and $x_{n, 2} \in P \cap\left(\Omega_{2}-\bar{\Omega}_{1}\right)$ such that $x_{n, 1}=T_{n} x_{n, 1}$ and $x_{n, 2}=T_{n} x_{n, 2}$.

An argument similar to that in the proof of (5.10)-(5.12) and (5.14) shows that

$\left\{x_{n, 1}(t)\right\},\left\{x_{n, 2}(t)\right\}$ are uniformly bounded on $[0,1]$,

$\left\{x_{n, 1}^{\prime}(t)\right\},\left\{x_{n, 2}^{\prime}(t)\right\}$ are uniformly bounded on $[0,1]$,

$\left\{x_{n, 1}(t)\right\},\left\{x_{n, 2}(t)\right\}$ are equicontinous on $[0,1]$,

and

$$
\left\{-x_{n, 1}^{\prime}(t)\right\},\left\{x_{n, 2}^{\prime}(t)\right\} \text { are equicontinuous on }[0,1] \text {, }
$$

which together with the Arzela-Ascoli Theorem guarantees the existence of a subsequence $\left\{n_{j}\right\}$ of $N_{0}$ and a function $x_{0,1} \in C^{1}[0,1]$ with $\lim _{j \rightarrow+\infty} x_{n_{j}, 1} \rightarrow x_{0,1}$ with

$$
x_{0,1}^{\prime}(0)=0
$$

and the existence of a subsequence $\left\{n_{k}\right\}$ of $N_{0}$ and a function $x_{0,2} \in C^{1}[0,1]$ with $\lim _{k \rightarrow+\infty} x_{n_{k}, 2} \rightarrow x_{0,2}$ with

$$
x_{0,2}^{\prime}(0)=0
$$

An argument similar to that in the proof of (5.16)-(5-17) shows that $x_{0,1}(t)$ and $x_{0,2}(t)$ are two different positive of $\operatorname{BVP}(1.1)-(1.2)$ with $\left\|x_{0,1}\right\|<R_{1}<\left\|x_{0,2}\right\|$.

Example 6.1. Consider the boundary value problems

$$
\begin{gathered}
x^{\prime \prime}+\mu\left[1+\left|x^{\prime}\right|^{a}+\left|x^{\prime}\right|^{-d}\right]\left[1+x^{b}\right]=0, \quad t \in(0,1) \\
x^{\prime}(0)=0, \quad x(1)=\int_{0}^{1} x(s) d A(s),
\end{gathered}
$$

with $d A(t)=\frac{1}{8} \sin 2 \pi t d t, 0<a<1, b>1,0<d$ and $\mu>0$. If

$$
\mu<\sup _{c \in(0,+\infty)} \frac{\int_{0}^{\left(1-c_{0}\right) c} \frac{s^{d}}{s^{d}+s^{a+d}+1} d s}{1+c^{b}}
$$

then $\operatorname{BVP}(6.2)-(6.3)$ has at least two different positive solutions $x_{0,1}, x_{0,2} \in$ $C^{1}[0,1] \cap C^{2}(0,1)$. 
It is easy to see that all conditions of Theorem 6.1 hold. Then, Theorem 6.1 guarantees that $\mathrm{BVP}(6.2)-(6.3)$ has at least two different positive solutions $x_{0,1}, x_{0,2} \in C^{1}[0,1] \cap C^{2}(0,1)$.

\section{Multiple positive solutions to $\operatorname{BVP}(1.1)-(1.2)$ with singularity at $x=0$ and $x^{\prime}=0$}

In this section our nonlinearity $f$ may be singular at $x=0$ and $x^{\prime}=0$ and $c_{0}=\int_{0}^{1}|d A(s)|$.

Theorem 7.1 Suppose $\left(C_{1}\right)-\left(C_{3}\right)$ hold and

$$
\begin{aligned}
& \sup _{c \in(0,+\infty)} \frac{\left(1-c_{0}\right) c}{I^{-1}\left(\|q\|_{0}\left[c h(c)+\int_{0}^{c} w(s) d s\right]\right)}>1, \text { where } \\
& I(z)=\int_{0}^{z} \frac{u}{r(u)+v(u)} d u, z \in(0,+\infty), I(+\infty)=+\infty,\|q\|_{0}=\max _{t \in[0,1]} q(t) .
\end{aligned}
$$

Then (1.1) has at least two positive solutions $x_{0,1}, x_{0,2} \in C^{1}[0,1] \cap C^{2}(0,1)$ with $x_{0,1}(t) \geq 0$ and $x_{0,2}(t)>0$ on $(0,1)$.

Proof. From (7.1) and the continuity of $I^{-1}$, choose an $R_{1}>0$, and a $\varepsilon>0$ with $\varepsilon<\frac{R_{1}}{2}$ with

$$
\frac{R_{1}\left(1-c_{0}\right)}{I^{-1}\left(I(\varepsilon)+\left(R_{1}+\varepsilon\right) h\left(R_{1}+\varepsilon\right)\|q\|_{0}+\|q\|_{0} \int_{0}^{R_{1}+\varepsilon} w(s) d s\right)}>1 .
$$

From $\left(C_{3}\right)$, choose a $R_{2}>\max \left\{R_{1}, \frac{R^{\prime}}{a}\right\}\left(R^{\prime}\right.$ is defined as in (2.12)).

Let $n_{0} \in\{1,2, \cdots\}$ be chosen so that $\frac{2}{n_{0}}<\varepsilon$, and let $N_{0}=\left\{n_{0}, n_{0}+1, \cdots\right\}$. For each $n \in N_{0}$, for $x \in P$, define

$\left(T_{n} x\right)(t)=\alpha[x]+\int_{0}^{1} G(t, s) q(s) f\left(s, x(s)+\frac{1}{n}(1-s)+\frac{1}{n}, x^{\prime}(s)-\frac{1}{n}\right) d s, \quad t \in[0,1]$.

Lemma 2.6 guarantees that $T_{n}: P \rightarrow P$ is continuous and compact (here $\gamma_{1}(t)=\frac{1}{n}(1-t)+\frac{1}{n}$ and $\gamma_{2}(t) \equiv-\frac{1}{n}$, for $\left.t \in[0,1]\right)$.

Set

$$
\Omega_{1}=\left\{x \in C_{p} \mid\|x\|<R_{1}\right\}
$$

and

$$
\Omega_{1}=\left\{x \in C_{p} \mid\|x\|<R_{2}\right\}
$$


An argument argument to that in the proof in Theorem 6.1 yields two different positive solutions $x_{0,1}(t)$ and $x_{0,2}(t)$ with $x_{0,1} \in \Omega_{1} \cap P$ and $x_{0,2} \in\left(\Omega_{2}-\bar{\Omega}_{1}\right) \cap P$.

Example 7.1. Consider the boundary value problems

$$
\begin{gathered}
x^{\prime \prime}+\mu\left[1+\left|x^{\prime}\right|^{a}+\left|x^{\prime}\right|^{-e}\right]\left[1+x^{b}+x^{-d}\right]=0, \quad t \in(0,1) \\
x^{\prime}(0)=0, \quad x(1)=\int_{0}^{1} x(s) d A(s),
\end{gathered}
$$

with $d A(t)=\frac{1}{8} \sin 2 \pi t d t, 0<a<1, b>1,0<d<1, e>0$ and $\mu>0$. If

$$
\mu<\sup _{c \in(0,+\infty)} \frac{\int_{0}^{\left(1-c_{0}\right) c} \frac{s^{e+1}}{s^{e}+s^{a+}+1} d s}{c+c^{b+1}+\frac{1}{1-d} c^{1-d}}
$$

then $\mathrm{BVP}(7.2)-(7.3)$ has at least two different positive solutions $x_{0,1}, x_{0,2} \in$ $C^{1}[0,1] \cap C^{2}(0,1)$.

It is easy to see that all conditions of Theorem 7.1 hold. Now Theorem 7.1 guarantees that $\operatorname{BVP}(7.2)-(7.3)$ has at least two different positive solutions $x_{0,1}, x_{0,2} \in C^{1}[0,1] \cap C^{2}(0,1)$.

\section{References}

[1] R.P. Agarwal and D.O'Regan, Existence theory for single and multiple solutions to singular positone boundary value problems, Jour. Differential Equations, 175(2001), 393-414.

[2] R.P. Agarwal and D.O' Regan, A survey of recent results for initial and boundary value problems singular in the dependent variable, Original Research Article Handbook of Differential Equations: Ordinary Differential Equations, 1(2000), 1-68.

[3] J.V. Baxley, A singular nonlinear boundary value problem: membrane response of a spherical cap, SIAM J. Appl. Math., 48(1988), 497-505.

[4] L.E. Bobisud, J.E. Calvert and W.D. Royalty, Some existence results for singular boundary value problems, Differential Integral Equations, 6 (1993), 553-571.

[5] K. Deimling, Nonlinear functional analysis, Springer Verlag, New York, 1985 . 
[6] D. Guo and V. Lakshmikantham, Nonlinear Problems in Abstract Cones, Academic Press, 1988.

[7] CH. P. Gupta, S. K. Ntouyas and P. CH. Tsamatos, Existence results for m-point boundary value problems, Differential Equations Dynam. Systems, 2(1994), 289-298.

[8] CH. P. Gupta and S. I. Trofimchuk, Solvability of a multi-point boundary value problem and related a priori estimates, Canad. Appl. Math. Quart., 6(1998), 45-60.

[9] L.Hu, Positive solutions for singular boundary value problems involving integral conditions, Boundary Value Problems, 2012(2012), 1-14

[10] V.A. Il'in, E.I. Moiseev, Nonlocal boundary value problems of the first kind for a SturmCLiouville operator in its differential and finite difference aspects, Differ. Equat., 23(1987), 803-810.

[11] V.A. Il'in, E.I. Moiseev, Nonlocal boundary value problem of the second kind for a Sturm-Liouville operator, Differ. Equat., 23(1987), 979C987.

[12] G. Infante and J. R. L. Webb, Positive solutions of some nonlocal boundary value problems, Abstr. Appl. Anal., 2003(2003), 1047-1060.

[13] C. Ji, B.Yan, Positive solutions for second-order singular three-poin boundary-value problems with sign-changing nonlinearities, Electronic Journal of Differential Equations, 2010(2010), 1-9.

[14] J. Jiang, L. Liu and Y. Wu, Positive Solutions for Second-Order Singular Semipositone Differential Equations Involving Stieltjes Integral Conditions, Abstract and Applied Analysis, 2012(2012), 1-21.

[15] G. L. Karakostas and P. CH. Tsamatos, Existence of multiple positive solutions for a nonlocal boundary value problem, Topol. Methods Nonlinear Anal., 19(2002), 109-121.

[16] G. L. Karakostas and P. CH. Tsamatos, Multiple positive solutions of some Fredholm integral equations arisen from nonlocal boundary-value problems, Electron. J. Differential Equations, 2002(2002), 1-17.

[17] R. Ma, Existence theorems for a second order m-point boundary value problem, J. Math. Anal. Appl., 211(1997), 545-555.

[18] R. Ma, Positive solutions of a nonlinear m-point boundary value problem, Comput. Math. Appl. 42(2001), 755-765. 
[19] D.O'Regan, Existence theory for nonlinear ordinary differential equations, Kluwer Acad. Publ., Dordrecht, 1997.

[20] S. Taliaferro, A nonlinear singular boundary value problem, Nonlinear Analysis, 3(1979), 897-904.

[21] J. R. L. Webb, Optimal constants in a nonlocal boundary value problem, Nonlinear Anal., 63(2005), 672-685.

[22] J. R. L. Webb and G. Infante, Positive Solutions of Nonlocal Boundary Value Problems: A Unified Approach, Oxford Journals Mathematics \& Physical Sciences Journal London Mathematical Society, 74(2006), 673693.

[23] J. R. L. Webb and K. Q. Lan, Eigenvalue criteria for existence of multiple positive solutions of nonlinear boundary value problems of local and nonlocal type, Topol. Methods Nonlinear Anal. 27 (2006), 91-115.

[24] J. R. L. Webb and G. Infante, Positive solutions of nonlocal boundary value problems involving integral conditions, Nonlinear Differential Equations and Applications, 15(2008), 45-67.

Baoqiang Yan,

Department of Mathematics,

Shandong Normal University,

Jinan, 250014, P.R.China.

Email: yanbqcn@aliyun.com

Donal O'Regan,

School of Mathematics, Statistics and Applied Mathematics,

National University of Ireland,

Galway, Ireland.

Email: donal.oregan@nuigalway.ie

Ravi P. Agarwal,

Department of Mathematics,

Texas A and M University-Kingsville,

Texas 78363, USA.

Email: Ravi.Agarwal@tamuk.edu 